\title{
Separation of anomalous and synchrotron emissions using WMAP polarization data
}

\author{
M.-A. Miville-Deschênes ${ }^{1,2}$, N. Ysard ${ }^{1}$, A. Lavabre ${ }^{1}$, N. Ponthieu ${ }^{1}$, J. F. Macías-Pérez ${ }^{3}$, \\ J. Aumont ${ }^{3,4}$, and J. P. Bernard ${ }^{4}$
}

\author{
${ }^{1}$ Institut d'Astrophysique Spatiale (CNRS), Université Paris-Sud, Bât. 121, 91405 Orsay, France \\ e-mail: mamd@ias.u-psud.fr \\ 2 Canadian Institute for Theoretical Astrophysics, University of Toronto, 60 St. George Street, Toronto, ON, M5S 3H8, Canada \\ 3 LPSC, Université Joseph Fourier Grenoble 1, CNRS/IN2P3, Institut National Polytechnique de Grenoble, 53 Av. des Martyrs, \\ 38026 Grenoble, France \\ ${ }^{4}$ Centre d'Étude Spatiale des Rayonnements, 9 Av. du Colonel Roche, 31028 Toulouse, France
}

Received 30 January 2008 / Accepted 10 September 2008

\begin{abstract}
Context. Apart from its contribution to cosmology, the WMAP data brings new information on the Galactic interstellar medium. In particular the polarization data provide constraints on the Galactic magnetic field and the synchrotron emission, while the intensity data can be used to study the anomalous microwave emission.

Aims. The main goals of this study is to use the information from both WMAP intensity and polarization data to do a separation of the Galactic components, with a focus on the synchrotron and anomalous emissions.

Methods. Our analysis is made at $23 \mathrm{GHz}$ where the signal-to-noise ratio is the highest and the estimate of the CMB map is less critical. Our estimate of the synchrotron intensity is based on an extrapolation of the Haslam $408 \mathrm{MHz}$ data with a spatially varying spectral index constrained by the WMAP $23 \mathrm{GHz}$ polarization data and a bi-symmetrical spiral model of the galactic magnetic field with a turbulent part following a $-5 / 3$ power law spectrum.

Results. The $23 \mathrm{GHz}$ polarization data are found to be compatible with a magnetic field with a pitch angle $p=-8.5^{\circ}$ and an amplitude of the turbulent part of the magnetic field 0.57 times the local value of the field, in agreement with what is found using rotation measures of pulsars and polarized extinction by dust. The synchrotron spectral index between $408 \mathrm{MHz}$ and $23 \mathrm{GHz}$ obtained from polarization data and our model of the magnetic field has a mean value of $\beta_{s}=-3.00$ with a limited spatial variation with a standard deviation of 0.06 . When thermal dust, free-free and synchrotron are removed from the WMAP intensity data, the residual anomalous emission is highly correlated with thermal dust emission with a spectrum in agreement with spinning dust models.

Conclusions. Considering a classical model of the large scale Galactic magnetic field, we show that the polarization data of WMAP are in favor of a soft synchrotron intensity highly correlated with the $408 \mathrm{MHz}$ data. Furthermore the combination of the WMAP polarization and intensity data brings strong evidence for the presence of unpolarized spinning dust emission in the 20-60 GHz range. In preparation for the Planck mission this joint analysis of polarization and intensity data opens new perspective on the study of the Galactic interstellar medium and on the component separation exercise.
\end{abstract}

Key words. ISM: magnetic fields - radio continuum: ISM - turbulence - polarization - dust, extinction

\section{Introduction}

One of the greatest challenges of observing the Cosmic Microwave Background (CMB) in the $20-200 \mathrm{GHz}$ range resides in the separation of the CMB and foreground emission. This task is facilitated by the fact that the intensity of the emission from the Galactic interstellar medium reaches a minimum in this range. On the other hand, even if the Galactic emission is weak, it is still stronger than the CMB over a significant fraction of the sky. In addition the identification of the cosmological signal is complicated by the fact that several interstellar emissions are superimposed in this frequency range: free-free, synchrotron, thermal dust. There are also strong evidence of an excess of emission in this range, discovered by Kogut et al. (1996b,a) in the COBE-DMR data. This so-called "anomalous emission" is remarkably well correlated with thermal dust emission at $100 \mu \mathrm{m}$ with a spectrum which can be confused with that of free-free or synchrotron emissions.
Leitch et al. (1997) showed that the correlation of this excess with $100 \mu \mathrm{m}$ extends to sub-degree scales in the North Celestial Loop (NCL) region. The lack of correlation of the anomalous emission with $\mathrm{H} \alpha$ measurements, also highlighted by de Oliveira-Costa et al. (2002) using Tenerife and COBEDMR data, led Leitch et al. (1997) to conclude that free-free emission can only account for the data if it comes from gas with $T_{\mathrm{e}}>10^{6} \mathrm{~K}$. Draine \& Lazarian (1998a) ruled out this interpretation based on energetic arguments and suggested rotational or spinning dust as a plausible emission mechanism (Draine \& Lazarian 1998b). This interpretation received strong support from dedicated ground observations (e.g. Watson et al. 2005; Fernandez-Cerezo et al. 2006) and from the analysis of the WMAP data (Lagache 2003; Finkbeiner 2004; Davies et al. 2006).

The WMAP team proposed an alternative explanation. Bennett et al. (2003a) and Hinshaw et al. (2007) argued that high-energy electrons, responsible for the synchrotron emission 
at WMAP frequencies, are produced in star forming regions which are also bright in the infrared, suggesting a natural correlation between synchrotron and dust emission. As the synchrotron emission is less correlated with dust emission at lower frequencies (e.g. $408 \mathrm{MHz}$ ), the dust correlated emission observed in the WMAP data is explained as a change in the synchrotron spectral index which can only be attributed to a local change of the energy distribution of cosmic rays. Spatial variations of the correlation between the magnetic field intensity and the gas density would not produce a frequency dependent behavior (see Sect. 3.4). This interpretation has one important drawback: the correlation between the $23 \mathrm{GHz}$ WMAP emission and the $100 \mu \mathrm{m}$ emission extends down to low column density cirrus clouds, where there is no star forming activities and no local production of cosmic rays (see Davies et al. 2006).

In this paper we want to put some constraints on the presence and properties of the anomalous emission in the WMAP frequency range, while making minimum assumptions about the emission mechanisms. The difficulty of this task comes from our lack of knowledge on the other components at these frequencies, mostly synchrotron. Anomalous emission has been measured to be very weakly polarized by Battistelli et al. (2006). This can be understood if the carriers of the spinning dust emission are small dust grains which are known not to produce any polarization from extinction measurements (e.g. Martin 2007). Free-free is also known to produce no polarization ${ }^{1}$. We therefore follow Finkbeiner (2004) and use polarization data to estimate the synchrotron emission. Based on this assumption and on a model of the Galactic magnetic field, we use the WMAP $23 \mathrm{GHz}$ polarization data to put realistic constraints on the synchrotron intensity and isolate the anomalous emission component.

The paper is organized as follow: the WMAP data we used are summarized in Sect. 2. Then Sect. 3 describes the models we used for the Galactic components, Sect. 4 describes our model for the synchrotron polarized data and for the Galactic magnetic field, and Sect. 5 presents the anomalous emission extracted from the WMAP data. The results are discussed and summarized in Sect. 6.

\section{WMAP data}

In this analysis we used the three-years WMAP products available in the Healpix pixelisation scheme (Gorski et al. 2005) on the LAMBDA website ${ }^{2}$. We used the temperature and polarization $(I, Q, U)$ coadded maps per frequency in the $K(23 \mathrm{GHz})$, $K$ a $(33 \mathrm{GHz}), Q(41 \mathrm{GHz}), V(61 \mathrm{GHz})$ and $W(94 \mathrm{GHz})$ bands. The original angular resolution of each map ranges from $0.23^{\circ}$ at $94 \mathrm{GHz}$ to $0.93^{\circ}$ at $23 \mathrm{GHz}$. The maps were all smoothed to a common resolution of $1^{\circ}$. We also used the Galactic Kp2 and point sources masks provided by the WMAP team.

To remove the Cosmic Microwave Background (CMB) from the data we used the estimate based on the Internal Linear Combination (ILC) method proposed by the WMAP team and applied on the three years data (Bennett et al. 2003a). The resolution of the ILC map is also $1^{\circ}$. As shown by Davies et al. (2006) and by Dobler \& Finkbeiner (2008) the estimate of the $\mathrm{CMB}$ can have a significant impact on the determination of the foreground spectra. In our case, it is a sub-dominant effect as our analysis is conducted at $23 \mathrm{GHz}$ where the Galaxy-to-CMB ratio is the highest.

\footnotetext{
${ }^{1}$ Expect at the edge of HII regions.

2 http://lambda.gsfc.nasa.org
}

By design WMAP is unable to measure the zero level at each frequency. This has no consequence for the study of the CMB fluctuations but it has an importance for the determination of the foreground properties. In practice there is a degeneracy between the zero level and the absolute foreground component amplitudes one can deduced from the data, unless one relies on a correlation analysis like Davies et al. (2006) who measured a mean spectral index of $\beta_{s} \approx-3$ between $408 \mathrm{MHz}$ and $23 \mathrm{GHz}$ at high Galactic latitude. In their analysis Eriksen et al. (2008) fitted jointly the CMB, foregrounds and zero level. In order to estimate the zero level they put a constrain on the average value of $\beta_{s}$ based on the results of Davies et al. (2006). In our analysis we rely on the zero level determined by Eriksen et al. (2008). In practice this zero level correction is only relevant at $23 \mathrm{GHz}$.

\section{Galactic components}

\subsection{WMAP data model}

In the following we consider that the WMAP intensity $I_{v}$ (corrected for $\mathrm{CMB}$, zero level and dipole, and smoothed at $1^{\circ}$ ) at frequency $v$ is the sum of free-free $\left(F_{v}\right)$, synchrotron $\left(S_{v}\right)$, thermal dust $\left(D_{v}\right)$ and anomalous emissions $\left(A_{v}\right)^{3}$ :

$I_{v}=S_{v}+F_{v}+D_{v}+\left[A_{v}\right]$.

In polarization, the model of the WMAP data involves less components as the free-free and anomalous emissions are assumed to be unpolarized. The CMB polarization level is so weak (Page et al. 2007) that it is not included in the data model. The polarized emission model is thus simply:

$P_{v}=S_{v}^{P}+D_{v}^{P}$

\subsection{Free-free}

Free-free emission is a rather difficult component of the interstellar emission to identify as it does not dominate at any frequency. $\mathrm{H} \alpha$, corrected for dust extinction, is often used as a proxy for free-free as they both depend linearly on the emission measure, $E M=\int n_{\mathrm{e}}^{2} \mathrm{~d} l$ (see Dickinson et al. 2003; Finkbeiner 2003). On the other hand, several systematic errors can affect the estimate of free-free emission from $\mathrm{H} \alpha$ corrected for extinction (see Bennett et al. 2003a; Finkbeiner 2004). Finkbeiner (2004) estimated that an error of a factor of 2 is likely in some parts of the sky solely due to an imperfect extinction correction. Electronic temperature variations are also expected, with lower values in the Galactic plane due to a higher metallicity and therefore more efficient cooling. Estimate of spatial variations of $T_{\mathrm{e}}$ are not available and a constant value on the sky is usually assumed.

It is important to point out that the free-free spectral index is a slowly varying function of frequency and electron temperature (Bennett et al. 1992; Dickinson et al. 2003; Bennett et al. 2003b):

$\beta_{\mathrm{ff}}=2+\frac{1}{10.48+1.5 \ln \left(T_{\mathrm{e}} / 8000 \mathrm{~K}\right)-\ln \left(v_{\mathrm{GHz}}\right)}$.

At $23 \mathrm{GHz}$, the free-free spectral index varies only from 2.13 to 2.16 for $4000<T_{\mathrm{e}}<10000 \mathrm{~K}$ and it is not affected by extinction. Based on the analysis by Davies et al. (2006) we also note that, at this frequency, the spectral index of free-free is significantly different than that of synchrotron $(\beta \sim-3)$ and anomalous emission $(\beta \sim-2.85)$.

\footnotetext{
${ }^{3}$ In one model (model \#1) we assume no anomalous emission.
} 


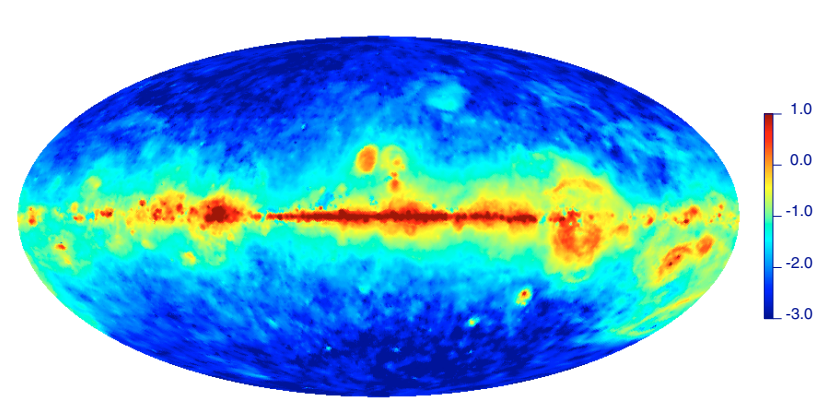

Fig. 1. Free-Free emission at $23 \mathrm{GHz}$ (in $\log _{10}(\mathrm{mK})$ ) estimated using $\mathrm{H} \alpha$ emission corrected for extinction (following Dickinson et al. 2003) and the WMAP MEM decomposition. For $E(B-V) \geq 2$, the free-free emission is the one obtained from the WMAP MEM decomposition. For $E(B-V)<2$, the free-free emission is the one estimated using $\mathrm{H} \alpha$ emission, except when the WMAP MEM free-free is lower.

Based on this physical property of the free-free emission Bennett et al. (2003b); Hinshaw et al. (2007) estimated the freefree emission in the WMAP data using MEM and considering a constant spectral index of 2.14 over the whole sky. In the following, we rely on the result of the MEM decomposition of Bennett et al. (2003b) for the estimate of the free-free emission in regions where $E(B-V)>2$ (or $A_{V} \geq 6$ ). The same assumption guided the work of Sun et al. (2008). In more diffuse regions we used the free-free estimate of Dickinson et al. (2003) based on a $\mathrm{H} \alpha$ all-sky template at $1^{\circ}$ resolution, unless the WMAP MEM free-free is lower than the $\mathrm{H} \alpha$ estimate. The free-free template we used at $23 \mathrm{GHz}$ is shown in Fig. 1.

\subsection{Thermal dust}

To estimate thermal dust emission in the WMAP data we used model \#7 of Finkbeiner et al. (1999), multiplied by 1.2 to account for the emission in the $94 \mathrm{GHz}$ band (Hinshaw et al. 2007). The correlation of the predicted thermal dust emission with $\left(I_{94}-F_{94}\right)$ at $94 \mathrm{GHz}$ and inside the Kp2 mask is 0.96 . We note that this component contributes only to $\sim 1 \%$ of the emission at $23 \mathrm{GHz}$ and that it does not affect our analysis of the synchrotron and anomalous emission at this frequency. This is true also in polarization as the polarization fraction of thermal dust is at the level of only a few percent (Benoit et al. 2004; Ponthieu et al. 2005; Page et al. 2007). Therefore at $23 \mathrm{GHz}$, where most of our analysis is done, we considered the polarized intensity to be entirely due to synchrotron emission: $P_{23}=S_{23}^{P}$.

\subsection{Synchrotron}

The synchrotron intensity depends on the cosmic ray (electron) density $\left(n_{\mathrm{e}}\right)$ and the strength of the magnetic field perpendicular to the line of sight $\left(B_{\perp}\right)$. For a cosmic ray distribution following a power law, $N(E) \propto E^{-s}$, the synchrotron intensity at frequency $v$ is:

$S(v)=\epsilon_{S}(v) \int_{z} n_{\mathrm{e}} B_{\perp}^{(1+s) / 2} \mathrm{~d} z$

where the integral is over the line of sight $z$ and $B_{\perp}=\sqrt{B_{x}^{2}+B_{y}^{2}}$ with the plane $x-y$ corresponding to the plane of the sky. The emissivity term $\epsilon_{S}(v)$ is given by a power law:

$\epsilon_{s}(v)=\epsilon_{0} v^{\beta_{s}}$ where

$\beta_{s}=-(s+3) / 2$.

It follows that the synchrotron intensity spectrum is a power law

$S(v)=S\left(v_{0}\right)\left(\frac{v}{v_{o}}\right)^{\beta_{s}}$.

For a typical value of cosmic ray spectrum $s=3$, we obtain $\beta_{s}=-3$.

The most reliable estimate of synchrotron emission to date is the $408 \mathrm{MHz}$ all-sky map of Haslam et al. (1982). In the following we estimate the synchrotron emission at any WMAP frequency using an extrapolation of the Haslam $408 \mathrm{MHz}$ map $\left(S_{408}\right)$ corrected for free-free emission as provided by the WMAP team:

$S_{v}=S_{408}\left(\frac{v}{0.408}\right)^{\beta_{s}}$

The extrapolation of the $408 \mathrm{MHz}$ emission at WMAP frequencies relies on an estimate of $\beta_{s}$ and of its variations on the sky. All-sky estimates of the synchrotron spectral index between $408 \mathrm{MHz}$ and $2326 \mathrm{MHz}$ have been attempted by Giardino et al. (2002) and Platania et al. (2003). They obtained compatible values of $\beta_{s}=-2.7 \pm 0.1$. On the other hand the spectral index varies with frequency (Banday \& Wolfendale 1991) due to electron aging effects. This effect is expected to be important in the WMAP range which makes uncertain the extrapolation to $23 \mathrm{GHz}$ of the spectral index defined at lower frequencies.

From the analysis of the WMAP data Finkbeiner (2004) found that a constant $\beta_{s}=-3.05$ between $408 \mathrm{MHz}$ and $23 \mathrm{GHz}$ corrects the most obvious synchrotron features seen both at $408 \mathrm{MHz}$ and $23 \mathrm{GHz}$ (i.e. Loop 1). This is in accordance with the work of Davies et al. (2006) who obtained $\beta_{s}=-3.01 \pm 0.04$ outside the Kp2 mask.

In the following we describe four different models of the synchrotron spectral index between $408 \mathrm{MHz}$ and $23 \mathrm{GHz}$. In all models we considered that thermal dust emission is negligeable at $23 \mathrm{GHz}\left(D_{23} \sim 0\right)$ and that the free-free $\left(F_{v}\right)$ is given by our model described in Sect. 3.2.

\subsubsection{Model 1}

Following Bennett et al. (2003a), this model assumes that the $23 \mathrm{GHz}$ emission is only synchrotron and free-free, giving an upper limit on the synchrotron spectral index:

$\beta_{s}=0.248 \log \left(\frac{I_{23}-F_{23}}{S_{408}}\right)$

where the multiplicative factor 0.248 is simply $1 / \log (23 / 0.408)$. This map of $\beta_{s}$ is shown in the top panel of Fig. 2. Using this simple method, which does not take into account anomalous emission, we obtain $\beta_{s}=-2.84 \pm 0.12$. The histogram of $\beta_{s}$ is shown in Fig. 3 (solid line). Taking into account anomalous emission will lower the contribution of synchrotron at $23 \mathrm{GHz}$ which will increase the value of $\beta_{s}$. This $\beta_{s}$ is similar to the one obtained by Bennett et al. (2003a). The differences are mainly due to the zero level correction we applied and to our slightly different free-free estimate. 

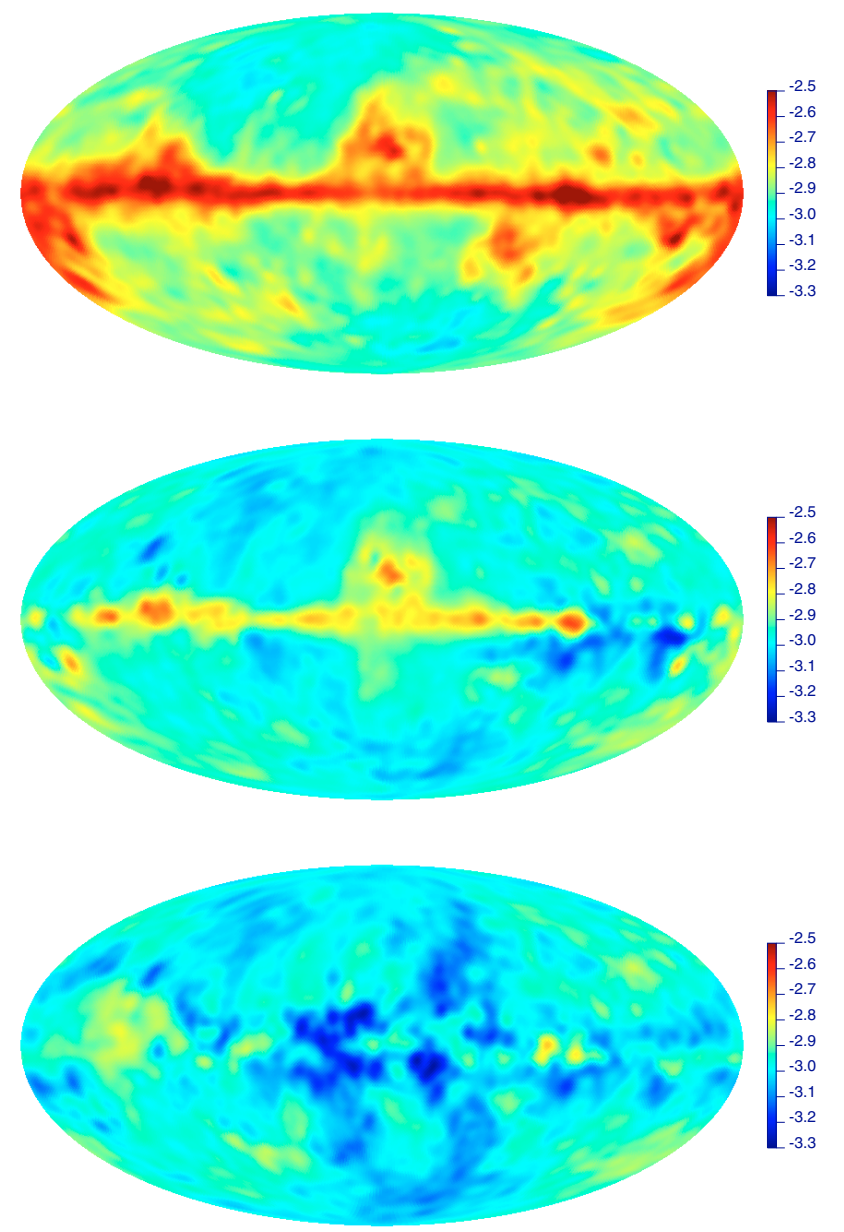

Fig. 2. Synchrotron spectral index $\left(\beta_{s}\right)$ between $408 \mathrm{MHz}$ and $23 \mathrm{GHz}$. All maps were smoothed at $5^{\circ}$. Top: model $1-$ this is making the assumption of no anomalous emission. The map is similar to the one of (Bennett et al. 2003b, their Fig. 4). Middle: model 3 - assumes a spinning dust component at $23 \mathrm{GHz}$ which scales with $E(B-V)$. Bottom: model $4-\beta_{s}$ obtained from polarization data for $p=-8.5^{\circ}, \chi_{0}=8^{\circ}$, $r_{0}=11^{\circ}$, and $\sigma_{\text {turb }}=1.7 \mu \mathrm{G}$.

\subsubsection{Model 2}

In this model we assume the presence of anomalous emission making no hypothesis on its spatial distribution and we set a constrain on $\beta_{s}$ considering it constant with a value of $\beta_{s}=-3$ in accordance with Davies et al. (2006):

$I_{23}=S_{408}\left(\frac{23}{0.408}\right)^{-3}+F_{23}+A_{23}$.

When this synchrotron estimate and our free-free model are subtracted, the residual $\left(A_{23}\right)$ is shown to be well correlated to the estimate of $E(B-V)$ of Schlegel et al. (1998) smoothed at 1 degree. In diffuse regions $(E(B-V)<0.5)$ we obtained $A_{23} \sim(0.65 \pm 0.01) E(B-V)+(0.03 \pm 0.01)$ (see Fig. 4$)$.

Even though the estimate of $E(B-V)$ of Schlegel et al. (1998) has been shown to overestimate systematically the extinction in regions where colder dust is present (see Cambrésy et al. 2001; Cambrésy et al. 2005), it remains a relatively accurate tracer of dust column density in diffuse regions, typically where $E(B-$ V) $<0.5$.

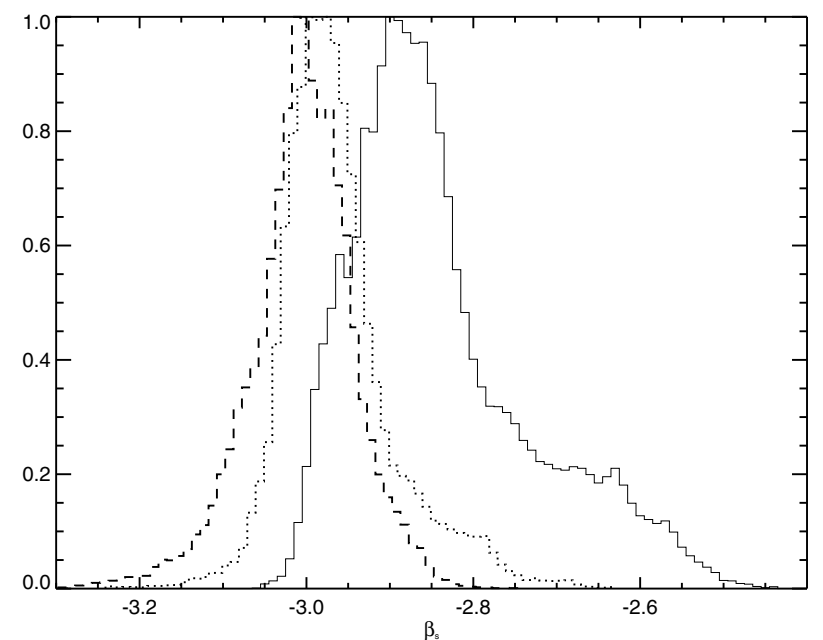

Fig. 3. Histogram of $\beta_{s}$ between $408 \mathrm{MHz}$ and $23 \mathrm{GHz}$ smoothed at $5^{\circ}$. solid line: model $1, \beta_{s}=-2.83 \pm 0.11$ dotted line: model $3, \beta_{s}=$ $-2.96 \pm 0.07$ dashed line: model $4, \beta_{s}=-3.00 \pm 0.06$.

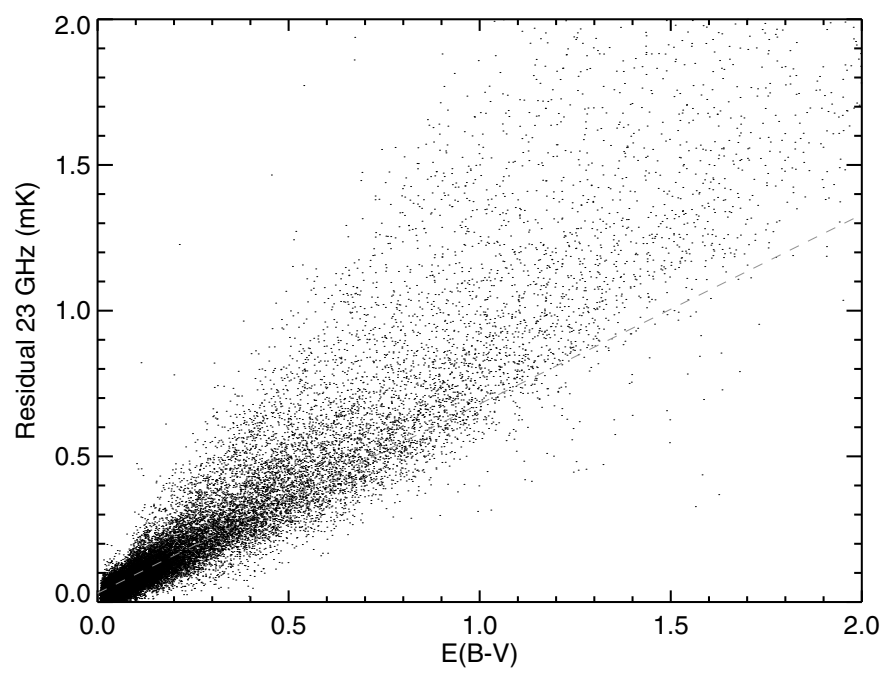

Fig. 4. Residual $23 \mathrm{GHz}$ emission when free-free, point sources and synchrotron with a constant $\beta_{s}=-3$ are subtracted, as a function of $E(B-V)$. The dashed line is $0.65 E(B-V)+0.03$.

\subsubsection{Model 3}

We can improve the estimate of $\beta_{s}$ and of its spatial variations by assuming a template for the anomalous emission component. Here we make the assumption that the anomalous emission is well correlated with dust column density (or $E(B-V)$. We added a component that scales with $E(B-V): I_{23}=S_{23}+F_{23}+a E(B-$ $V)$. Here the synchrotron spectral index is:

$\beta_{s}=0.248 \log \left(\frac{I_{23}-F_{23}-a E(B-V)}{S_{408}}\right)$.

In this simple model we use $a=0.65 \mathrm{mK} / \mathrm{mag}$ which is the slope observed in diffuse regions in model 2 (see Fig. 4). Compare to model 1, this model (see Fig. 2, middle panel and Fig. 3 (dotted line)) produces a steeper $\beta_{s}$ on average $\left(\beta_{s}=-2.96 \pm 0.07\right)$ but compatible with model 2 (constant $\beta_{s}=-3$ ) which is expected as we used the value of $a$ deduced from model 2. Most importantly the spatial variations of $\beta_{s}$ is reduced by a factor of 2 compared to model 1 . This has to be appreciated considering that some spatial variations of $\beta_{s}$ could be due to spatial variations of the anomalous emission that we considered to be strictly 
proportional to $E(B-V)$. Nevertheless the fact that taking into account an extra component reduces significantly the variations of $\beta_{s}$ is a strong indication of a better modeling of the data. This is compatible with gamma ray observations from which Strong et al. (2000) do not predict strong spatial variations of the synchrotron spectral index, mostly due to the large interaction length of cosmic ray electrons.

\subsubsection{Model 4}

In order to separate the synchrotron and anomalous emissions at $23 \mathrm{GHz}$ we would like to have an estimate of $\beta_{s}$ independent of intensity measurements at $23 \mathrm{GHz}$ and of any constrains on the spatial variation of the anomalous emission. In model 4 we used the fact that the polarized intensity at $23 \mathrm{GHz}$ is dominated by synchrotron. Based on a model of the Galactic magnetic field we estimated the synchrotron intensity at $23 \mathrm{GHz}$ using the WMAP polarization data. This model is described in the next section.

\section{Synchrotron polarization at $23 \mathrm{GHz}$}

\subsection{Synchrotron polarization}

Similarly to the synchrotron intensity (see Eq. (4)) the Stokes parameters $Q$ and $U$ of polarized synchrotron emission integrated along the line of sight are

$Q(v)=f_{s} \epsilon_{s}(v) \int_{\mathrm{z}} n_{\mathrm{e}} B_{\perp}^{(1+s) / 2} \cos 2 \phi \sin \alpha \mathrm{d} z$

and

$U(v)=f_{s} \epsilon_{s}(v) \int_{\mathrm{z}} n_{\mathrm{e}} B_{\perp}^{(1+s) / 2} \sin 2 \phi \sin \alpha \mathrm{d} z$

where

$\cos 2 \phi=\frac{B_{x}^{2}-B_{y}^{2}}{B_{\perp}^{2}}$

$\sin 2 \phi=\frac{-2 B_{x} B_{y}}{B_{\perp}^{2}}$

and

$\sin \alpha=\sqrt{1-B_{z}^{2} / B^{2}}$

The polarization fraction $f_{s}$ is related to the cosmic ray energy distribution slope $s$ :

$f_{s}=\frac{s+1}{s+7 / 3}$.

For a typical value of cosmic ray spectrum $s=3$ we obtain $f_{s}=0.75$.

The polarized intensity is simply

$P(v)=\sqrt{Q(v)^{2}+U(v)^{2}}$

and the polarization angle

$\gamma(v)=0.5 \tan ^{-1}\left(\frac{U(v)}{Q(v)}\right)$.
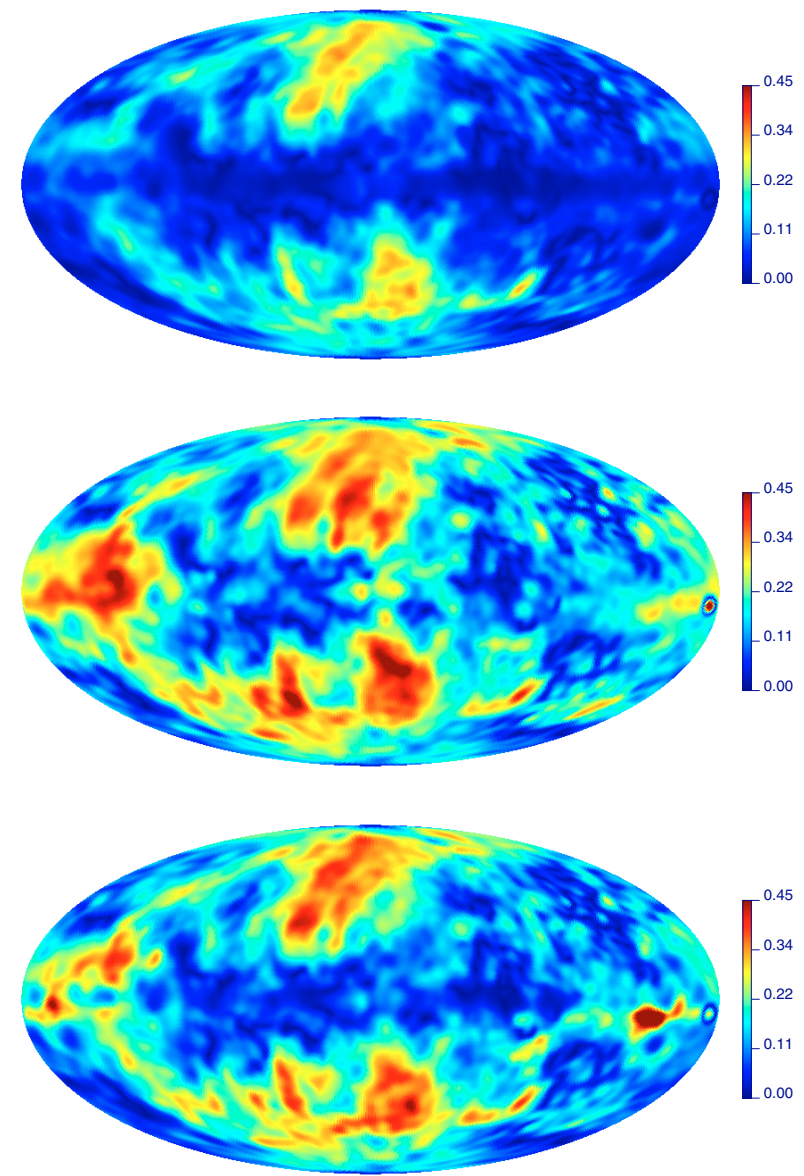

Fig. 5. Polarization fraction (from 0 to 1 ) at $5^{\circ}$ resolution. The polarization $P$ is from the WMAP $K$ band data and the intensity $S$ is from models 1 to 3 (from top to bottom).

\subsection{Polarization fraction at $23 \mathrm{GHz}$}

Combining Eqs. (4), (12) and (13), the polarization fraction is given by the following simple equation:

$\frac{P(v)}{S(v)}=f_{s} g$

where $f_{s}$ is the intrinsic polarization fraction related to the cosmic rays energy spectrum (see Eq. (17)) and $g$ is a geometrical reduction factor that reflects the depolarization due to the variation of the angle between the magnetic field and the line of sight directions. The $g$ factor depends on the structure of the magnetic field and on the distribution of the cosmic rays in threedimensions (i.e. the integrals in Eqs. (4), (12) and (13)). It takes values between 0 and 1 ; it reaches a maximum when the magnetic field is parallel to the plane of the sky $\left(B=B_{\perp}\right)$. In practice it never reaches 1 as the magnetic field direction fluctuates on any line of sight, due to both the spiral structure of the magnetic field and turbulent motions.

We can estimate the polarization fraction at $23 \mathrm{GHz}$ using the WMAP polarization data $\left(P_{23}\right)$ and estimates of the synchrotron intensity $\left(S_{23}\right)$ given by models 1 to 3 . The corresponding polarization fraction maps, smoothed at $5^{\circ}$ are shown in Fig. 5. The polarization fraction for model 1 is similar to the one given by (Kogut et al. 2007, their Fig. 4). Models 2 and 3 gives very similar polarization fraction maps, which is expected considering that both models have almost the same average $\beta_{s}$ and considering the small dispersion of $\beta_{s}$ in model 3. On the other hand the 
polarization fraction of model 1 is systematically lower than in other models because of the assumption of no anomalous emission which increases the synchrotron intensity (and not its polarization). Apart from the average level, the most significant difference between the polarization fraction of model 1 to 3 is the large scale structure. Models 2 and 3 shows less polarization in the inner galaxy than towards the anti-center region. Model 1 has a systematically lower polarization value in the plane over all longitudes without a clear raise of the polarization fraction towards the anti-center. The contrast between low and mid latitudes is also stronger in model 1 than in models 2 and 3.

The structures seen in the polarization fraction maps are due to real depolarization structure (due to the magnetic field 3D structure - large scale and turbulent components) but also to our imperfect estimate of $\beta_{s}$. Nevertheless it is remarkable that the polarization fraction given by models 2 and 3 are very similar even with important differences in the definition of $\beta_{s}$. In the following we look for a magnetic field model that allows to reproduce these polarization fraction maps.

\subsection{Model of polarization at $23 \mathrm{GHz}$}

In model 4 we used the relation between intensity and polarization to estimate the synchrotron intensity from the $23 \mathrm{GHz}$ polarization data which are assumed to be dominated by synchrotron polarization:

$S_{23} \sim \frac{P_{23}}{g f_{s}}$.

The synchrotron spectral index is then given by:

$\beta_{s}=0.248 \log \left(\frac{P_{23}}{g f_{s} S_{408}}\right)$.

This relies on a proper estimate of the reduction factor $g$. The construction of $g$ depends on a model of the large scale galactic magnetic field and of its fluctuations due to turbulence. The latter will have a strong impact on the depolarization value, especially in the Galactic plane where lines of sight are longer.

In order to estimate $g$, we used the fact that $g \sim P / S\left(f_{s}\right.$ is almost constant - see Eq. (17)). To estimate the polarization fraction $P / S$ we use Eqs. (4), (12), (13) and (18) which depends on the structure of the magnetic field $B$ in three dimensions and on the electronic density $n_{\mathrm{e}}$. Following Page et al. (2007) we used a variation of the cosmic ray electron density with galactocentric radius and height:

$n_{\mathrm{e}}=n_{0} \exp \left(-r / h_{r}\right) \operatorname{sech}^{2}\left(z / h_{z}\right)$

with $h_{r}=5 \mathrm{kpc}$ and $h_{z}=1 \mathrm{kpc}$. Sun et al. (2008) adopted a similar description of the cosmic ray distribution.

\subsubsection{Galactic magnetic field at large scales}

Following what is found in the analysis of pulsar rotation measures (RM, Han et al. 2006) we modeled the Galactic magnetic as a bi-symmetrical spiral (BSS):

$B(r, \theta, z)=B_{0}(r) \cos \left(\theta-\psi \ln \frac{r}{r_{0}}\right) \cos (\chi)$

where $\psi=1 / \tan (p), p$ being the pitch angle and $\chi(z)=$ $\chi_{0} \tanh (z / 1 \mathrm{kpc})$ describing the vertical $(z)$ component of the magnetic field (see Page et al. 2007). Using RMs at high Galactic latitudes, Han et al. (2006) estimated that the amplitude of this

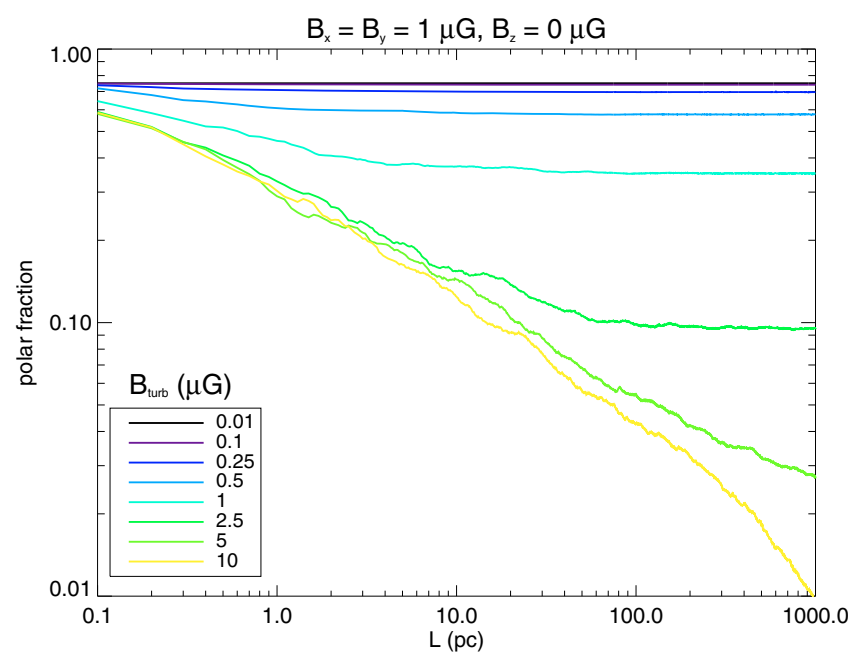

Fig. 6. Polarization fraction as a function of depth along the line of sight. The large scale magnetic field is perpendicular to the line of sight, giving maximum polarization ( $g=1$ and $\left.f_{s}=0.75\right)$ with no turbulence. The different curves give the level of polarization we would observe for increasing level of the turbulent component of the magnetic field.

component of the magnetic field is almost 10 times smaller than the horizontal components. We found that $\chi_{0}$ has little impact on the modeling.

Rotation measures are compatible with a slight variation of the magnetic field strength $B_{0}$ with Galactic radius $R$, modeled by Han et al. (2006) with a slowly rising exponential towards the Galactic center. In fact this increase is not very clear and the data are also compatible with $B_{0}$ constant with $R$. In this analysis we found that a constant $B_{0}$ provides a better fit to the WMAP data.

\subsubsection{Turbulent part of the magnetic field}

The large scale spiral structure of the Galactic magnetic field is disrupted locally by turbulent motions of the gas. The amplitude of the perturbation will depend on the turbulence energy density; on average data indicates that the energy in the turbulent part of the magnetic field is of the same order as the energy in the large scale field (see Beck 2001, and references therein). In addition Minter \& Spangler (1996) showed that the power spectrum of the turbulent part of the magnetic field follows a power law with an exponent of $-5 / 3$ at scales smaller than 100 pc. At larger scales, the structure of the magnetic field is dominated by the spiral pattern (Han et al. 2004).

The impact of this turbulent part of the magnetic field on polarization is to lower the polarization fraction with respect to what would be observed with only the large scale magnetic field. This turbulent depolarization is stronger at low Galactic latitudes as lines of sight in the Galactic plane cross more fluctuations than at high latitudes. This effect is shown in Fig. 6 where we have simulated the effect of a turbulent magnetic field superposed on a large scale field perpendicular to the line of sight $\left(B_{x}=B_{y}=1 \mu \mathrm{G}\right.$ and $\left.B_{z}=0 \mu \mathrm{G}\right)$. The $x, y$ and $z$ components of the turbulent field are independent 1D Gaussian random fields along the line of sight ( 10000 points, cell size of $0.1 \mathrm{pc}$ ) with a power spectrum following a $-5 / 3$ power law at scales lower than $100 \mathrm{pc}$. For this exercise we assumed a constant cosmic ray density and $s=3$ (i.e. $f_{s}=0.75$ ). The polarization fraction is then computed by integrating numerically Eqs. (4), (12) and (13) for increasing values of the depth $L$. This was repeated for increasing values of $B_{\text {turb }}$ from 0.01 to $10 \mu \mathrm{G}$. 

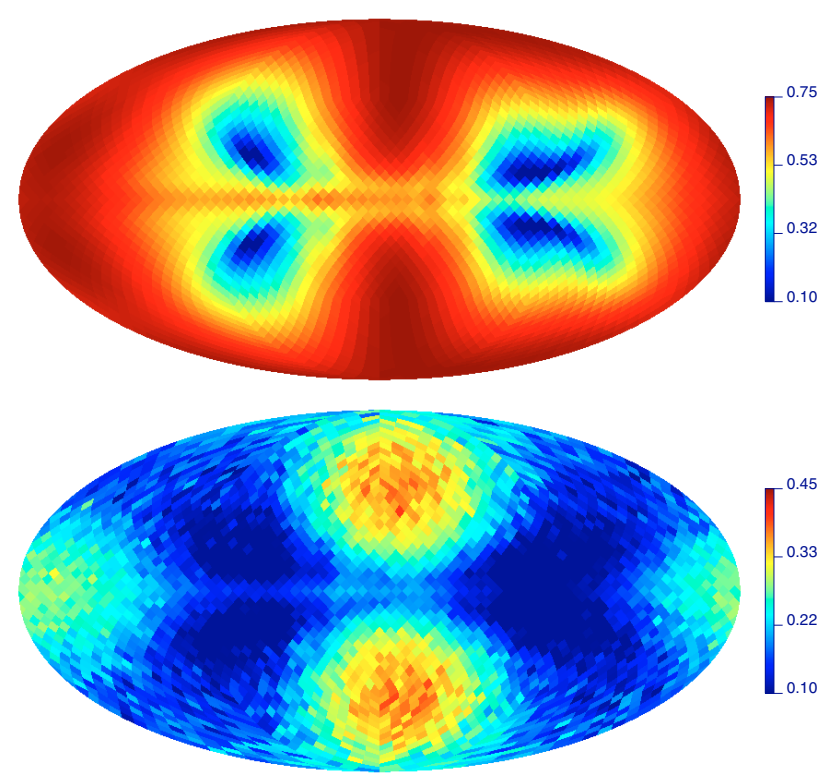

Fig. 7. Model of the polarization fraction with (bottom) and without (top) turbulence. Parameters of the magnetic field model are $p=-8.5^{\circ}$, $\chi_{0}=8^{\circ}, r_{0}=11 \mathrm{kpc}, B_{0}=3 \mu \mathrm{G}$ and turbulence of $\sigma_{\text {turb }}=1.7 \mu \mathrm{G}$.

As expected the polarization fraction decreases generally with the depth of the line of sight and the amplitude of the turbulent motions. More and/or stronger turbulent fluctuations reduce the coherence of the field and the polarization fraction. In fact the behavior observed here depends solely on the ratio of $B_{\text {turb }} / B$ and not on their respective absolute values. We noted that for moderate values of $B_{\text {turb }} / B$, the polarization fraction reaches an asymptotic value. This values is reached at larger line of sight depths for greater $B_{\text {turb }} / B$ values.

The map of $g$ was computed by adding a turbulent vector field to the BSS magnetic field model for each sky coordinate. Therefore every lines of sight are independent and no coherence on the plane of the sky due to turbulence is introduced. The turbulent part added here allows to set the proper average level of $g$ but it also modifies the structure of $g$ as the depolarization due to turbulence depends on the length of the line of sight and the ratio of $B_{\perp} / B$. Nevertheless most of the structure of $g$ is set by the spiral structure of the field.

An example of a $g f_{s}$ map, with and without turbulence added is shown in Fig. 7. Depolarization due to turbulence is increased in the galactic plane (where line of sight are longer) and in regions where $B_{\perp} / B$ is lower. The two bright spots above and under the Galactic center corresponds to regions where the Galactic magnetic field is almost perpendicular to the line of sight.

\subsubsection{Fitting the magnetic field parameters}

Unlike Page et al. (2007) we did not fit the parameters of the model on the polarization angle which is very sensitive to high latitudes structures like Loop 1. Instead the parameters are determined by fitting the polarization fraction map directly. Our model is not depedant on the absolute values of $B_{0}$ and $B_{\text {turb }}$ but only to their ratio. The parameters we are varying to fit the data are: $p, r_{0}, \chi_{0}$ and $B_{\text {turb }}$. The fixed parameters are: $\gamma_{\text {turb }}=-5 / 3$, $l_{\text {max }}=100 \mathrm{pc}, B_{0}=3 \mu \mathrm{G}$ and $n_{\mathrm{e}}$.

The fitting of the parameters is done by minimizing the $\chi^{2}$ of the polarization fraction of our Galactic magnetic field model with respect to the polarization fraction deduced from models 2
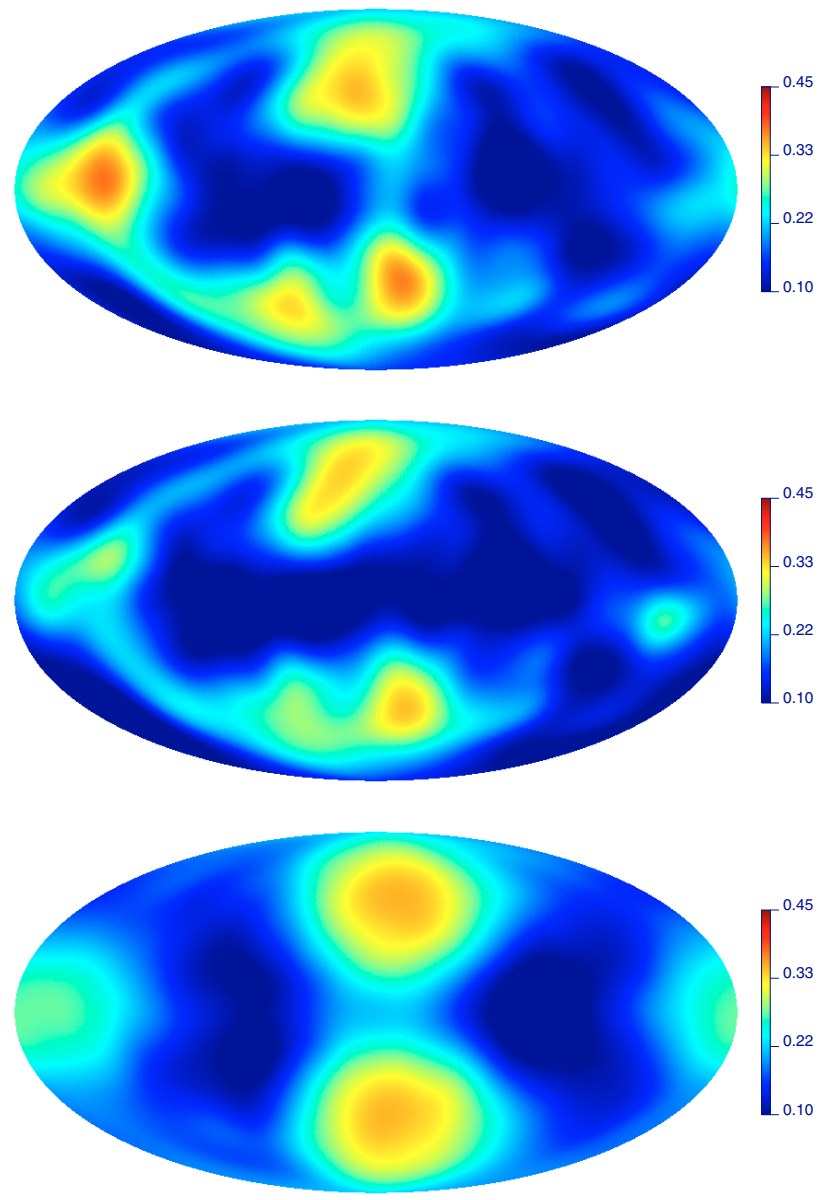

Fig. 8. Polarization fraction smoothed at $20^{\circ}$. Top: model 2: constant $\beta_{s}=-3$. Middle: model 3: $\beta_{s}$ estimated with a anomalous emission proportional to $E(B-V)$. Bottom: model 4: $\beta_{s}$ estimated from polarization for $p=-8.5^{\circ}, \chi_{0}=8^{\circ}, r_{0}=11 \mathrm{kpc}, B_{0}=3 \mu \mathrm{G}$ and turbulence of $\sigma_{\text {turb }}=1.7 \mu \mathrm{G}$.

and 3 and smoothed at $20^{\circ}$ (see Fig. 8). Similar values are found for both model 2 and 3. The best fit model gives $p=-8.5^{\circ}$, $\chi=8^{\circ}, r_{0}=11.0 \mathrm{kpc}, B_{\mathrm{turb}} / B_{0}=0.57$. The polarization fraction of our best fit model is shown in Fig. 8. The correlation factor are 0.65 and 0.72 for model 2 and 3 respectively.

The parameters found for the large scale field are in very good agreement with what is obtained from the analysis of pulsar rotation measures and from a previous analysis of the WMAP data by Jansson et al. (2007). In particular the pitch angle value we found $\left(p=-8.5^{\circ}\right)$ is remarkably close to the values obtained using pulsar rotation measures (Han \& Qiao 1994; Han et al. 1999; Indrani \& Deshpande 1999; Beck 2001; Han et al. 2006) or polarized starlight absorption measurements (Heiles et al. 1996) where values are in found in the range $-7<p<-11^{\circ}$. On the other hand our result is not compatible with the result of Page et al. (2007) who obtained $p=-35^{\circ}$.

The values of $B_{\text {turb }} / B_{0}$ found for model 2 and 3 is also remarkably close to the one obtained from the analysis of the synchrotron emission (Phillipps et al. 1981; Beck 2001) which gives $B_{\text {turb }} / B_{0} \sim 0.66$, which implies energy equipartition between the turbulent and large scale components. Our result is also in agreement with the work of Jones et al. (1992) who obtained $B_{\text {turb }} / B_{0} \sim=0.6$ using polarized extinction measurements at $2.2 \mu \mathrm{m}$. On the other hand, and similarly to Sun et al. (2008) who did not consider the presence of anomalous emission at $23 \mathrm{GHz}$, 

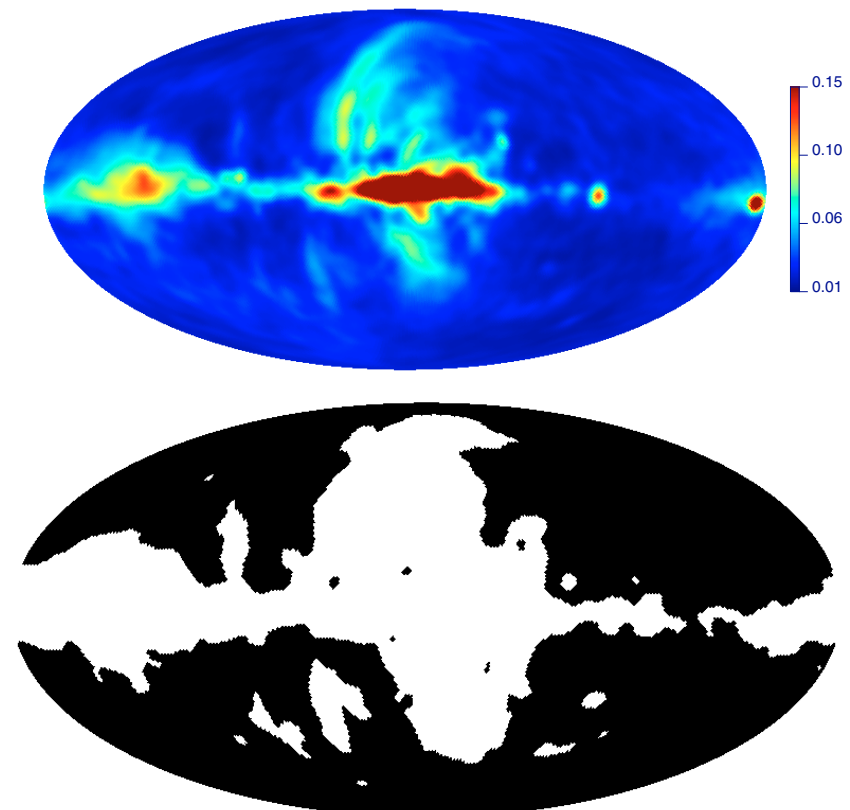

Fig. 9. Top: polarized intensity (in $\mathrm{mK}$ ) at $23 \mathrm{GHz}$, smoothed at $5^{\circ}$. Bottom: mask showing regions of signal-to-noise ratio greater than 3 .

model 1 requires $B_{\text {turb }} / B_{0} \sim 1.5$ to fit the data, which is significantly stronger than the value obtained by Phillipps et al. (1981); Beck (2001). This is due to the fact that anomalous emission contributes a significant fraction of the intensity at $23 \mathrm{GHz}$ and that it is not polarized. If (unpolarized) anomalous emission is not taken into account at $23 \mathrm{GHz}$, a stronger depolarization (and therefore more turbulence) is needed to account for the low polarization fraction. The incapacity of model 1 to reproduce the right level of $B_{\text {turb }} / B_{0}$ is a strong evidence for the presence of an unpolarized anomalous emission at $23 \mathrm{GHz}$. Therefore in the following we will not consider model 1.

\subsection{Model of synchrotron emission}

We build the synchrotron spectral index using

$\beta_{s}=\frac{\log \left(M_{23} / S_{408}\right)}{\log (23 / 0.408)}$

where $M_{23}$ is the model of the intensity at $23 \mathrm{GHz}$ obtained from polarization:

$M_{23}=\frac{P_{23}}{g f_{s}}$.

$P_{23}$ is the polarization map from WMAP smoothed at $5^{\circ}$ (shown in Fig. 9), $f_{s}$ is given by Eq. (17) and the depolarization fraction $g$ is given by our model, smoothed at $20^{\circ}$ to remove any small scale fluctuations introduced by the turbulent part of the magnetic field.

A reliable estimate of $M_{23}$ can only be made for pixels where the signal-to-noise ratio of the polarization data is significant. We defined a mask shown in Fig. 9 which gives regions with polarization signal-to-noise ratio large enough to estimate the synchrotron intensity. Inside this mask $\beta_{s}$ was estimated using Eq. (25). Outside the polarization mask, $E(B-V)$ is mostly lower than 1 which corresponds to regions of the sky where the anomalous emission correlates well with extinction. Therefore we used the estimate of $\beta_{s}$ given by model 3 in regions outside the polarization mask. The resulting $\beta_{s}$ map at a resolution of $5^{\circ}$ is shown
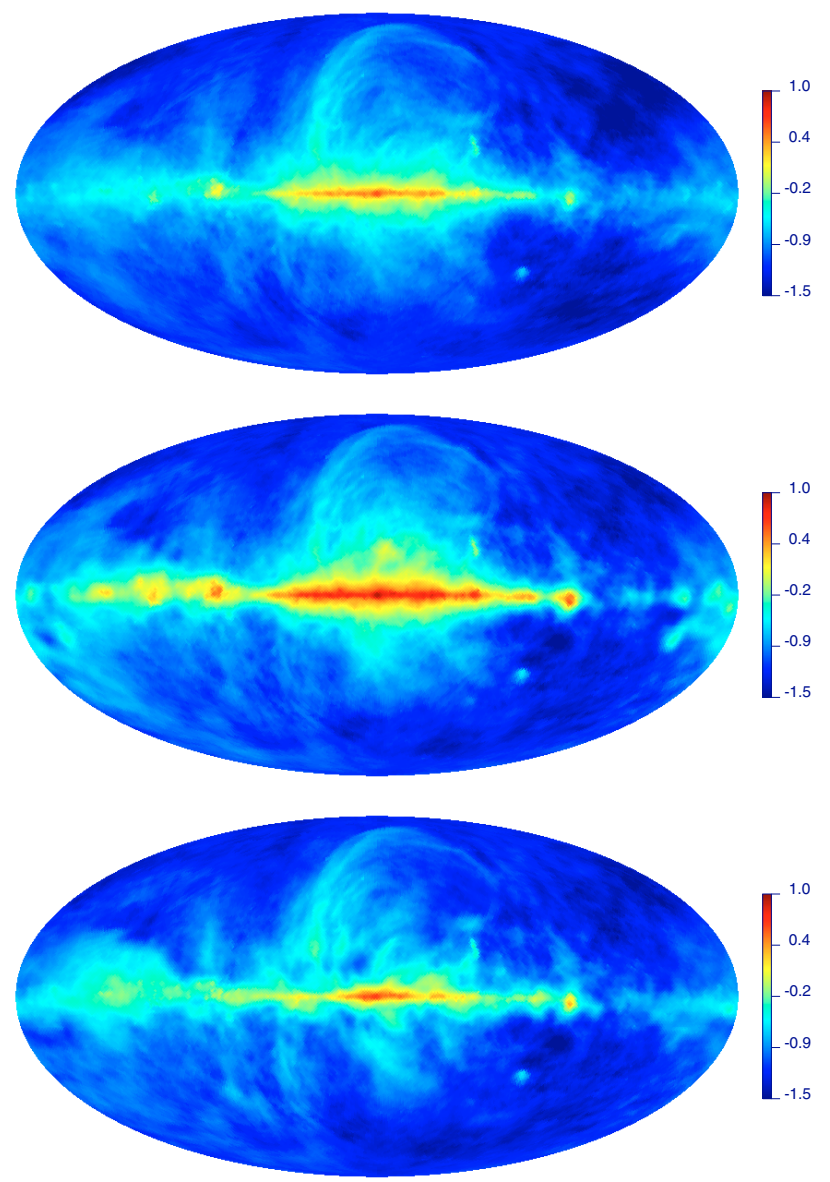

Fig. 10. Synchrotron emission (in $\log _{10}(\mathrm{mK})$ ) at $23 \mathrm{GHz}$ for models 2 (top), 3 (middle) and 4 (bottom). The synchrotron map for model 2 (top) is a scaling of the $408 \mathrm{MHz}$ map with a constant $\beta_{s}=-3$.

in the bottom panel of Fig. 2. Even if $\beta_{s}$ was estimated with two different models, we note that there are no striking discontinuities at the boundaries of the mask. Compared to models 1 and 3 the galactic plane does not stand out in this spectral index map. In that respect the spectral index map deduced from the polarization data is much more similar to the ones determined between 408, 1420 and $2326 \mathrm{MHz}$ by Giardino et al. (2002) and by Platania et al. (2003).

The histogram of $\beta_{s}$ is shown in Fig. 3 (dashed line). It has an average value of -3.00 and a standard deviation of 0.06 . The minimum dispersion of $\beta_{s}$ is thus obtained for model 4.

\section{Anomalous emission in the WMAP data}

The predicted synchrotron intensity at $23 \mathrm{GHz}$ for models 2,3 and 4 is shown in Fig. 10. For model 3 (Fig. 10-middle), there is more synchrotron emission in the plane than for the two other models. This is due to the spectral index used which is significantly shallower in the plane (see Fig. 2-middle). The synchrotron emission for models 2 and 4 are very similar in overall intensity but there are significant spatial variations, especially in the Galactic plane, partly due to the fact that model 4 does not include spatial variations of $g$ at scales smaller than $20^{\circ}$.

The residual emission, when free-free and synchrotron are removed from the $23 \mathrm{GHz}$ data, are shown in Fig. 11 for models 2, 3 and 4. We attribute this residual to the anomalous emission. The anomalous emission obtained for models 2 (constant 

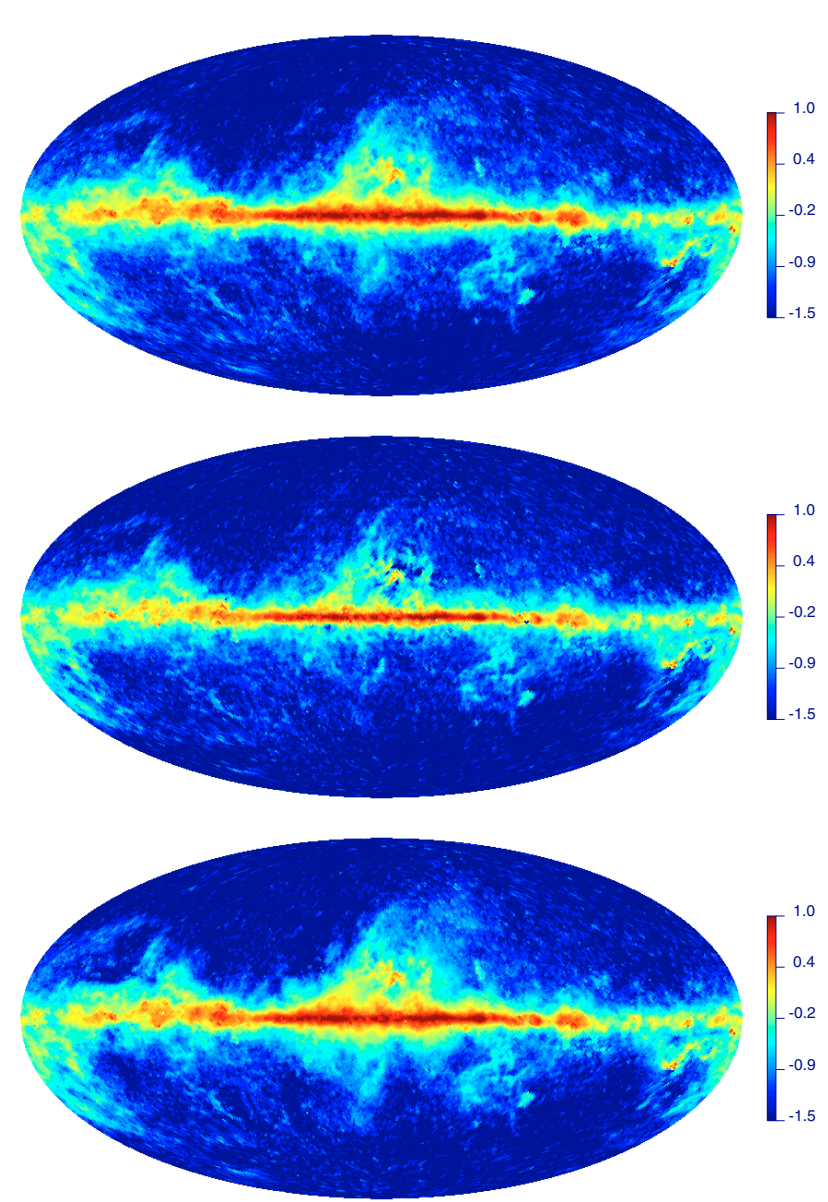

Fig. 11. Estimate of the anomalous emission (in $\left.\log _{10}(\mathrm{mK})\right)$ at $23 \mathrm{GHz}$ for models 2 (top), 3 (middle) and 4 (bottom).

$\left.\beta_{s}=-3\right)$ and model 4 ( $\beta_{s}$ obtained from polarization) are very similar. The difference between these two models is well fitted by a Gaussian of average $0.002 \mathrm{mk}$ and $\sigma=0.015 \mathrm{mK}$. For both models, the correlation factor of the anomalous emission with extinction $(E(B-V))$ is 0.94 . This analysis shows that the synchrotron emission at $23 \mathrm{GHz}$ can be rather well estimated, to first approximation, with an extrapolation of the $408 \mathrm{MHz}$ with a constant spectral index $\beta_{s}=-3$. In the following we describe the properties of the anomalous emission for model 4 .

The anomalous emission is dominating the signal from the Galactic plane to mid-latitude regions, with a mean intensity up to ten times higher than synchrotron (see Fig. 12). On the other hand, we note that the average level of synchrotron emission at high latitude is about three times that of the anomalous emission, likely due to the large scale height of cosmic ray electrons. The Gould belt's cloud have strong anomalous emission and known high-latitude cirrus regions like the North Celestial Loop are also seen in anomalous emission.

The anomalous emission was also extracted at 33, 41 and $61 \mathrm{GHz}$ using the synchrotron spectral index map of model 4 . The emissivity per hydrogen atom at each frequency of the anomalous emission was estimated by correlating it with $N_{\mathrm{H}}\left(\mathrm{cm}^{-2}\right)=5.8 \times 10^{21} E(B-V)\left(\right.$ see Fig. 13) for $N_{\mathrm{H}}>$ $10^{21} \mathrm{~cm}^{-2}$. The spectrum obtained using model 4 is shown in Fig. 13; the anomalous emission spectrum is the same within $1 \%$ for models 2 and 4. In Fig. 13 the anomalous emission spectrum is compared to the spinning dust model of Draine \& Lazarian (1998b) for the Warm Neutral Medium (WNM) which provides

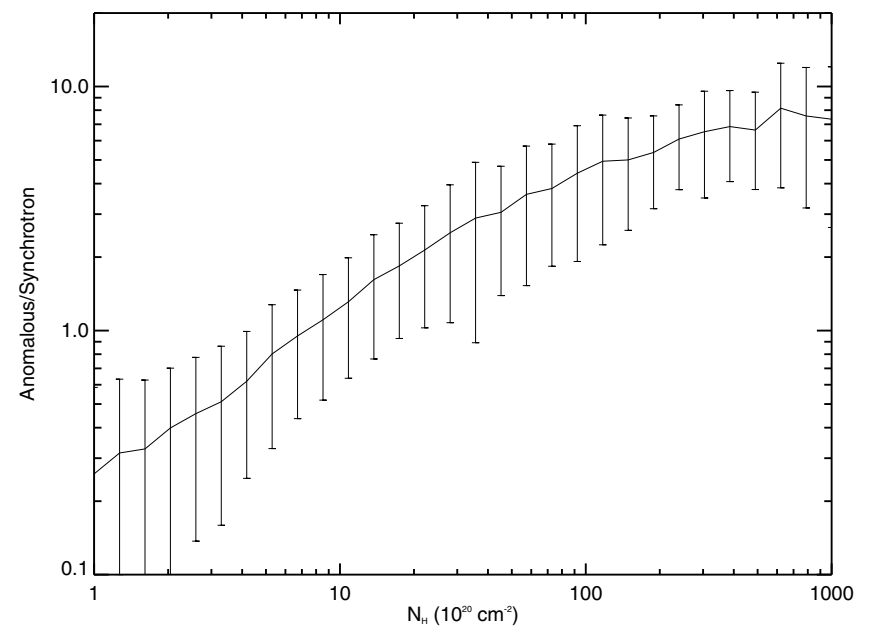

Fig. 12. Ratio of anomalous emission to synchrotron at $23 \mathrm{GHz}$ (model 4) as a function of column density. In the Galactic plane the anomalous is on average several times higher than the synchrotron emission. In the most diffuse regions at high Galactic latitude the synchrotron emission overcomes the anomalous emission in total intensity. The error bars represent the standard deviation of the anomalous to synchrotron ratio in each bin of column density.

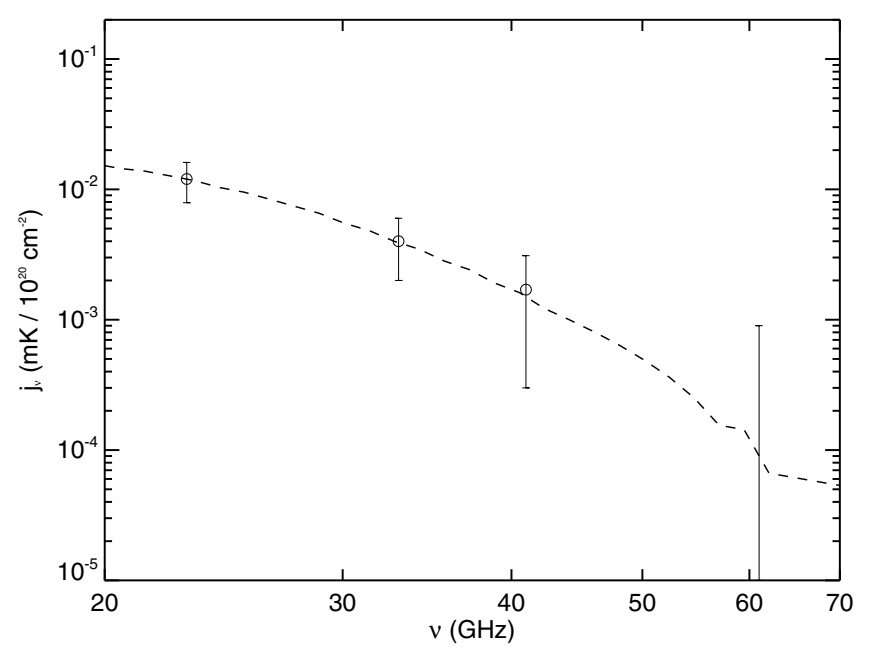

Fig. 13. Spectrum of the anomalous emissivity obtained by correlating the anomalous from model 4 with $N_{\mathrm{H}}=5.8 \times 10^{21} E(B-V)$. The correlation was estimated for $N_{\mathrm{H}}>10^{21} \mathrm{~cm}^{-2}$. The uncertainty is the standard deviation of the emissivity observed for the selected pixels. The emissivity at $61 \mathrm{GHz}$ is compatible with zero. The dashed curve is the emissivity spectrum, normalized at $23 \mathrm{GHz}$, given by Draine \& Lazarian (1998b) for the WNM.

a good fit to the data. A detailed analysis of the anomalous emission spectrum is left to a further study.

\section{Conclusion}

In this paper we presented a new analysis of the Galactic emission at $23 \mathrm{GHz}$ as observed by WMAP. The main goal of this study was to combine the total intensity and polarization data from WMAP to separate the synchrotron emission and the anomalous emission at this frequency. Our analysis is based on the hypothesis that 1) the $23 \mathrm{GHz}$ intensity data of WMAP is dominated by $\mathrm{CMB}$, synchrotron, free-free and anomalous emission; 2) the $23 \mathrm{GHz}$ polarization data is dominated by synchrotron only; 3 ) the average spectral index of synchrotron between $408 \mathrm{MHz}$ and $23 \mathrm{GHz}$ is close to $\beta_{s}=-3$, and 4) the 
Galactic magnetic field has a BSS large scale structure with a turbulent part that follows a $-5 / 3$ power spectrum at scales smaller than $100 \mathrm{pc}$.

We used the WMAP $23 \mathrm{GHz}$ polarization data to constrain the parameters of the magnetic field. The data are found compatible with a pitch angle of $-8.5^{\circ}$ and a ratio of the turbulent to regular part of the magnetic field of $B_{\text {turb }} / B_{0}=0.57$. The parameters of our Galactic magnetic field model are in good agreement with what is found with pulsar rotation measures, radio synchrotron emission and polarized extinction in the near-infrared. One key result of this analysis is that the equipartition between the turbulent and regular part of the magnetic field is only obtained if unpolarized anomalous emission is included in the modelling of the $23 \mathrm{GHz}$ data.

Based on our Galactic magnetic field model and the WMAP polarization data, we produced a map of the synchrotron spectral index in total intensity, between $408 \mathrm{MHz}$ and $23 \mathrm{GHz}$. The map of $\beta_{s}$ we found does not show a strong Galactic plane feature, in accordance with what was found in the radio by Giardino et al. (2002); Platania et al. (2003). Extrapolating the Haslam et al. $408 \mathrm{MHz}$ map with this new estimate of the synchrotron spectral index, we could separate the contribution of the synchrotron and anomalous emission at 23,33, 41 and $61 \mathrm{GHz}$.

The all-sky anomalous emission at $23 \mathrm{GHz}$ is found to be correlated to $E(B-V)$ (or to $A_{v}$ ) with a correlation factor of $94 \%$. It is well detected in the Galactic plane, in Gould Belt's clouds and in the main high-latitude cirrus clouds. In the Galactic plane the anomalous emission is up to ten times stronger than the synchrotron but it is only one third of the synchrotron in the most diffuse regions of the sky where the synchrotron, while very smooth, has an average level stronger than the anomalous emission, possibly due to the larger scale height of cosmic ray electrons compare to interstellar dust. Under the hypothesis that the synchrotron spectral index does not vary with frequency over the WMAP range, we found that the anomalous emission has a spectrum from 23 to $61 \mathrm{GHz}$ in accordance with the model of spinning dust from Draine \& Lazarian (1998b). A deeper analysis of the origin of the anomalous emission and of its consequences regarding the properties of interstellar dust will be the subject of a forthcoming paper.

The presence in the $20-200 \mathrm{GHz}$ range of several diffuse Galactic components with similar intensities and some spatial correlation makes the extraction of the CMB a complex task. In order to achieve sufficient accuracy on the cosmological signal the component separation needs to take advantage of the knowledge on the properties of diffuse Galactic emission. In this paper we have shown that combining temperature and polarization data helps to improve our model of Galactic emissions. The higher signal-to-noise ratio, the higher angular resolution, the larger frequency coverage and the increase number of frequency bands of the Planck data will also certainly help to make progress in this area.
Acknowledgements. Some of the results in this paper have been obtained using the HEALPix package (Gorski et al. 2005). This work was partly supported by the Canadian Space Agency.

\section{References}

Banday, A. J., \& Wolfendale, A. W. 1991, MNRAS, 248, 705

Battistelli, E. S., Rebolo, R., Rubino-Martin, J. A., et al. 2006, ApJ, 645, L141

Beck, R. 2001, Space Sci. Rev., 99, 243

Bennett, C. L., Smoot, G. F., Hinshaw, G., et al. 1992, ApJ, 396, L7 Bennett, C. L., Halpern, M., Hinshaw, G., et al. 2003a, ApJS, 148, 1 Bennett, C. L., Hill, R. S., Hinshaw, G., et al. 2003b, ApJS, 148, 97 Benoit, A., Ade, P., Amblard, A., et al. 2004, A\&A, 424, 571

Cambrésy, L., Boulanger, F., Lagache, G., \& Stepnik, B. 2001, A\&A, 375, 999

Cambrésy, L., Jarrett, T. H., \& Beichman, C. A. 2005, A\&A, 435, 131

Davies, R. D., Dickinson, C., Banday, A. J., et al. 2006, MNRAS, 758, 758

de Oliveira-Costa, A., Tegmark, M., Finkbeiner, D. P., et al. 2002, ApJ, 567, 363

Dickinson, C., Davies, R. D., \& Davis, R. J. 2003, MNRAS, 341, 369

Dobler, G., \& Finkbeiner, D. P. 2008, ApJ, 680, 1222

Draine, B. T., \& Lazarian, A. 1998a, ApJ, 494, L19

Draine, B. T., \& Lazarian, A. 1998b, ApJ, 508, 157

Eriksen, H. K., Dickinson, C., Jewell, J. B., et al. 2008, ApJ, 672, L87

Fernandez-Cerezo, S., Gutierrez, C. M., Rebolo, R., et al. 2006, MNRAS, 370, 15

Finkbeiner, D. P. 2003, ApJS, 146, 407

Finkbeiner, D. P. 2004, ApJ, 614, 186

Finkbeiner, D. P., Davis, M., \& Schlegel, D. J. 1999, ApJ, 524, 867

Giardino, G., Banday, A. J., Gorski, K. M., et al. 2002, A\&A, 387, 82

Gorski, K. M., Hivon, E., Banday, A. J., et al. 2005, ApJ, 622, 759

Han, J. L., \& Qiao, G. J. 1994, A\&A, 288, 759

Han, J. L., Manchester, R. N., \& Qiao, G. J. 1999, MNRAS, 306, 371

Han, J. L., Ferriere, K., \& Manchester, R. N. 2004, ApJ, 610, 820

Han, J. L., Manchester, R. N., Lyne, A. G., Qiao, G. J., \& van Straten, W. 2006, ApJ, 642, 868

Haslam, C. G. T., Salter, C. J., Stoffel, H., \& Wilson, W. E. 1982, A\&AS, 47, 1 Heiles, C., Reach, W. T., \& Koo, B. C. 1996, ApJ, 466, 191

Hinshaw, G., Nolta, M. R., Bennett, C. L., et al. 2007, ApJS, 170, 288

Indrani, C., \& Deshpande, A. A. 1999, New Astron., 4, 33

Jansson, R., Farrar, G. R., Waelkens, A., \& Ensslin, T. A. 2007, Large scale magnetic field of the Milky Way from WMAP3 data, [arXiv: 0708.2714]

Jones, T. J., Klebe, D., \& Dickey, J. M. 1992, ApJ, 389, 602

Kogut, A., Banday, A. J., Bennett, C. L., et al. 1996a, ApJ, 460, 1

Kogut, A., Banday, A. J., Bennett, C. L., et al. 1996b, ApJ, 464, L5

Kogut, A., Dunkley, J., Bennett, C. L., et al. 2007, ApJ, 665, 355

Lagache, G. 2003, A\&A, 405, 813

Leitch, E. M., Readhead, A. C. S., Pearson, T. J., \& Myers, S. T. 1997, ApJ, 486, L23

Martin, P. G. 2007, in Polarization 2005, ed. M.-A. Miville-Deschênes, \& F. Boulanger, EAS Publ. Ser., 23, 165

Minter, A. H., \& Spangler, S. R. 1996, ApJ, 458, 194

Page, L., Hinshaw, G., Komatsu, E., et al. 2007, ApJS, 170, 335

Phillipps, S., Kearsey, S., Osborne, J. L., Haslam, C. G. T., \& Stoffel, H. 1981, A\&A, 98, 286

Platania, P., Burigana, C., Maino, D., et al. 2003, A\&A, 410, 847

Ponthieu, N., Macías-Pérez, J. F., Tristram, M., et al. 2005, A\&A, 444, 327

Schlegel, D. J., Finkbeiner, D. P., \& Davis, M. 1998, ApJ, 500, 525

Strong, A. W., Moskalenko, I. V., \& Reimer, O. 2000, ApJ, 537, 763

Sun, X. H., Reich, W., Waelkens, A., \& Enßlin, T. A. 2008, A\&A, 477, 573

Watson, R. A., Rebolo, R., Rubiño-Martín, J. A., et al. 2005, ApJ, 624, L89 